Article

\title{
Vers une Amélioration de la Prestation de Services de Santé pour les Franco-Ontariens
}

\author{
Pascale-Anne M. Doucet, MPH(candid.); Robyn Gorham, EdD; Elena Hunt, PhD* \\ Université Laurentienne, Sudbury, Ontario, Canada. \\ *Auteur correspondant :ehunt@laurentian.ca \\ Reçu : ; accepté : ; Publié :
}

\section{Résumé}

Malgré le statut de dualité linguistique du Canada, la communauté francophone continue d'être laissée pour compte par les autorités du pays. Plus spécifiquement, l'étendue et l'accès restreints des services de santé offerts aux francophones en Ontario posent un grave danger pour cette population. Les établissements qui dispensent des services de santé continuent d'ignorer les politiques de soins existantes. Cette situation impose plusieurs actions au niveau fédéral, législatif et communautaire nécessaires afin d'assurer la garantie bilingue promise à la population canadienne. Parmi celles-ci, il faut augmenter la présence francophone dans les groupes décisionnels pour assurer la conception et l'établissement de programmes et services de santé axés sur les besoins uniques et distincts des franco-ontariens. Par ailleurs, l'engagement financier fédéral envers des organismes communautaires francophones est essentiel afin de fournir plus de services de santé en français, surtout dans les communautés nordiques et rurales de la province. Finalement, la subvention gouvernementale de futures recherches sur la santé des francophones est nécessaire puisque celles-ci vont servir de base solide pour déterminer comment mieux desservir cette population. Pour y arriver, il faudrait considérer le lobbying, l'encouragement des institutions qui dispensent des services de santé d'obtenir leur désignation bilingue, et la mise à jour de la Loi sur les services en français. Cependant, en continuant l'inaction, les Franco-Ontariens risquent de devenir assimilés au restant de la population, de dépérir et de voir leur santé se détériorer. Cet article cherche notamment à signaler la nécessité d'améliorer la prestation de services de santé en français en Ontario.

\begin{abstract}
Despite Canada's linguistic duality status, the francophone community continues to be neglected by the country's authorities. More specifically, the limited scope and access to health services offered to francophones in Ontario pose a serious threat to this population. Facilities providing health services continue to ignore existing health care policies. This situation imposes several actions at the federal, legislative and community levels necessary to ensure the bilingual guarantee promised to the Canadian population. Among these, we must increase the
\end{abstract}


Francophone presence in decision-making groups to ensure the design and establishment of health programs and services focused on the unique and distinct needs of Franco-Ontarians. Moreover, federal financial commitment to Francophone community organizations is essential in order to provide more health services in French, especially in northern and rural communities in the province. Finally, government funding for future research on the health of Francophones is necessary since this will serve as a solid basis for determining how to better serve this population. To achieve this, we would have to consider lobbying, encouraging institutions that provide health services to obtain their bilingual designation, and updating the French Language Services Act. However, by continuing inaction, Franco-Ontarians risk becoming assimilated into the rest of the population, wasting away and seeing their health deteriorating. This article seeks in particular to point out the need to improve the delivery of health services in French in Ontario.

Mots-clés: Francophone, services de santé, population minoritaire, Ontario, milieu rural

Keywords : Francophones, Health services, Minorities, Ontario, Rural

\section{Introduction}

Depuis plusieurs décennies, la quête d'équité et de justice sociale demeure un débat persistant au sein des diverses communautés francophones au Canada, malgré le statut officiel diglossique du pays. La société canadienne semble rapidement oublier que le français, nonseulement constitue la langue d'accueil de plusieurs nouveaux-arrivants, mais est aussi la langue d'un des deux peuples fondateurs du pays. Plus particulièrement, $l^{\prime}$ accès aux services de santé en français se maintient difficilement, surtout au nord de l'Ontario où se retrouvent les proportions les plus élevées de francophones ${ }^{[1\}}$. Ce n'est pas le manque de politiques gouvernementales à ce sujet qui cause des soucis, mais plutôt le fait que les politiques ne sont pas respectées par les divers institutions et établissements qui dispensent des services de soins de santé ${ }^{\{2\}}$. Ce problème sérieux met en danger le bien-être des francophones, et s'amplifie dans les communautés nordiques de l'Ontario ${ }^{11}$.

La province de l'Ontario est l'une des plus grandes et la plus peuplée parmi les treize provinces et territoires canadiens, comptant presque $40 \%$ de la population du pays ${ }^{[3\}}$. Divisée informellement en 2 régions distinctes, la province de l'Ontario comprend le Sud de l'Ontario, plus urbain et arable, et le Nord de
l'Ontario, plus vaste, principalement rural et moins cultivable, avec un terrain plus aride et rocheux. Le territoire nord-ontarien comprend presque $90 \%$ de celui de la province, mais seulement $6 \%$ de sa population. Selon le dernier recensement, en 2016, la population francoontarienne comptait au-dessus de 622.000 personnes, concentrée dans le nord-est de la province ${ }^{\{3\}}$.

L'accès restreint à des services de santé en français nuit à la garantie de services bilingues promis par les gouvernements, un problème sur lequel il est nécessaire de se pencher plus profondément, en raison de son impact sur la santé des minorités francophones. Ce texte cherche justement à explorer l'origine, la création et l'implémentation de la politique des services en santé en français, sa situation actuelle et les lacunes correspondantes quant aux services de santé offerts en français aux communautés du nord de l'Ontario, ainsi qu'à proposer des mesures possibles dans le but de remédier à cette situation.

\section{Contexte historique}

Dès 1969, le Canada adopte officiellement la première Loi sur les deux langues officielles, soit le français et l'anglais, visant à assurer aux Canadiens l'accès à des services de la part 
d'institutions fédérales dans l'une des deux langues officielles de leur choix. Cependant, certaines provinces ont décidé d'adopter leurs propres politiques linguistiques puisque la loi ne concernait que les institutions fédérales ${ }^{44\}}$.

En 1986, l'Ontario a officiellement adopté sa première Loi sur les services en français, qui n'entrait en vigueur que trois ans plus tard ${ }^{\{5\}}$. Cette loi stipule la désignation de 23 endroits spécifiques (villes, municipalités et districts) obligés d'offrir des services bilingues, en autant qu'ils comptent un minimum de 5000 résidents francophones ou bien une population ayant une présence francophone d'au moins $10 \%$ [5].

Toutefois, l'introduction de cette loi en Ontario n'a pas été acceptée par toutes les municipalités de la province. En effet, plusieurs villes telles que ThunderBay et Sault-Sainte Marie se sont opposées à la loi, en se déclarant comme étant des entités monolingues anglophones, malgré le fait que la loi ne poussait aucune exigence supplémentaire de leur part ${ }^{[5]}$. En réponse à cette situation, les Franco-Ontariens ont tenté de baptiser Ottawa en tant que ville officiellement bilingue, campagne qui n'a toujours pas atteint son but ${ }^{45}$. Par conséquent, il demeure toujours un écart significatif entre les services reçus par les francophones comparativement aux services reçus par les anglophones ${ }^{\{1\}}$.

La communauté franco-ontarienne minoritaire s'est d'ailleurs accoutumée au combat constant pour le simple droit de recevoir des services dans leur langue maternelle.

\section{L'exemple de l'Hôpital Montfort}

L'Hôpital Montfort, le seul hôpital francophone à l'Ouest du Québec, province canadienne majoritairement francophone, porte le drapeau d'avant-gardisme pour les francophones. Établi dès la fin des années 1940, cet hôpital fut conçu pour offrir des services en français à la communauté francophone grandissante dans les environs de la capitale nationale ${ }^{[6\}}$. Son ouverture fut possible grâce à la collaboration des chefs de file francophones d'Eastview, les Pères Montfortains, la congrégation des Filles de la Sagesse ainsi que plusieurs directeurs de l'Association canadiennefrançaise d'éducation de l'Ontario (ACFÉO) ${ }^{[6]}$.

En plus des services de santé francophones offerts à Montfort, les Filles de la Sagesse ont également fondé divers programmes de formation pour infirmières, techniciennes en radiologie et techniciennes de laboratoire, tous offerts directement à l'hôpital. En outre, l'affiliation officielle avec l'Université d'Ottawa en 1961 solidifie l'impact de l'hôpital envers la formation de futurs professionnels de la santé francophones en Ontario ${ }^{[6\}}$.

La menace de fermeture de l'hôpital en 1997 par le gouvernement provincial a provoqué plusieurs révoltes de la communauté francophone, menant ainsi au mouvement renommé "SOS Montfort" dirigé par Gisèle Lalonde ${ }^{17\}}$. Ce fut la première lutte de telle envergure éprouvée par les Franco-Ontariens depuis le débat contre le Règlement 17, lorsque la francophonie dans les écoles bilingues en Ontario fut interdite ${ }^{[6]}$. À vrai dire, cinq ans d'activisme politique ardant et de nombreux litiges légaux ont enfin mené à la victoire de la communauté francophone, et les portes de l'Hôpital Montfort sont toujours ouvertes. Non seulement que cet événement probant a soutenu l'identité des franco-ontariens, mais il a aussi confirmé la protection que la Charte canadienne des droits accorde à la minorité linguistique francophone en Ontario [7]. Bref, cette victoire marquante est devenue un symbole significatif pour la société francophone, mais ne change pas le fait que cette communauté doit continuer de mener un combat sans répit afin d'obtenir et garder des droits équitables. L'événement Montfort fut révélateur devant les franco-ontariens, face à leur pouvoir en tant que communauté, mais aussi face à leur vulnérabilité persistante lorsque comparée à la population majoritaire ${ }^{\{2\}}$.

\section{Situation actuelle}


À l'époque contemporaine, les deux dernières décennies ont apporté quelques améliorations menant vers de meilleurs services pour les francophones au Canada. Parmi ceux-ci, citons : le développement du plan d'action pour la dualité linguistique canadienne, la naissance du Consortium national de formation en santé (CNFS) et de la Société Santé en français (SSF), ainsi que la reconnaissance de la langue en tant que déterminant de la santé de la part des subventionnaires de recherche. Il faut également noter l'arrivée du premier Réseau interdisciplinaire de recherche sur la santé des francophones en situation minoritaire (RISF), qui tient compte des disparités dans l'offre des services de santé aux francophones $\{8\}$ (p. 10-18).

Plusieurs politiques de soins furent établies par le gouvernement en guise d'assurer et de promettre une équité linguistique. À vrai dire, la Loi sur les services en français qui existe en Ontario est dite de garantir aux Franco-Ontariens la prestation des services en français [4\}. Néanmoins, malgré l'existence de cette Loi, le fait demeure qu'il existe un écart substantiel entre l'état des services de santé en anglais et celui en français en Ontario ${ }^{\{2\}}$. Ceci pourrait être lié au fait que la Loi sur les services en français est entrée en vigueur il y a au-delà de 30 ans et n'a reçu aucune mise à jour, en dépit des efforts de l'ancien commissaire aux services en français, François Boileau, qui fut baptisé le "chien de garde des francophones" en raison de son rôle, de

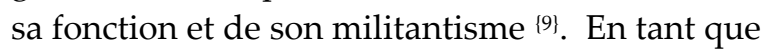
commissaire, M. Boileau avait "un mandat spécifique de veiller à la préservation et à la protection des droits linguistiques d'une communauté minoritaire" ${ }^{199}$. Effectivement, les responsabilités de $\mathrm{M}$. Boileau allaient au-delà du traitement de plaintes par rapport aux services en français. Il représentait la communauté francophone en faisant de la promotion et de la sensibilisation aux besoins des francophones, en fournissait des recommandations auprès du gouvernement afin d'assurer l'avancement des dossiers reliés à la francophonie. Pourtant, l'abolition récente de son poste en 2017 par le gouvernement conservateur signifie que l'ombudsman provincial est maintenant devenu responsable des plaintes concernant les FrancoOntariens ${ }^{19}$. Donc, toutes inquiétudes au sujet des services en français en Ontario ne seront plus prioritaires comme elles l'étaient avant, surtout puisque l'ombudsman a plusieurs autres demandes à répondre. Ceci est surtout alarmant en raison de la hausse en matière de plaintes des franco-ontariens qui fut témoignée l'an dernier. Effectivement, 435 plaintes furent soumises en 2018-2019, une augmentation de 38\% comparativement à l'année précédente ${ }^{[9]}$. Les Franco-Ontariens craignent que leurs soucis ne soient pas redressés, et que leur voix au niveau gouvernemental soit éteinte ${ }^{\{9\}}$.

Bref, le manque de prestations de soins en français demeure l'une des plus grandes menaces contre la santé des Franco-Ontariens. En Ontario, tout organisme ministériel provincial, tel que les hôpitaux, les foyers pour personnes âgées, et les services d'aide à l'enfance peuvent postuler pour une désignation officielle en tant qu'organisme offrant des services en français ${ }^{\{10\}}$. Toutefois, un rapport mené par l'Institut Canadien de Recherche sur les Minorités Linguistiques (ICRML), illustre avec précision l'état réel des services de santé en français hors Québec \{11\}. Ce rapport révèle que la majorité des hôpitaux n'offrent pas des services dans les deux langues officielles. Plus accablant est le fait que seulement la moitié des hôpitaux en Ontario offrent des services d'interprétation au public ${ }^{\{11\}}$. En milieu rural, où il existe déjà une pénurie globale de professionnels de la santé, les services de santé bilingues sont pratiquement inexistants \{11\}. En guise d'exemple, une étude menée en 2013 illustre la distribution précaire de médecins aptes à délivrer des soins en français en Ontario. Selon l'étude de Timony et al.\{12\}, la majorité des médecins pouvant offrir des services en français travaillent dans les communautés les moins francophones de la province et le taux de médecins francophones dans les communautés ontariennes considérées pauvrement francophones est de 5.6 médecins francophones par 1000 personnes francophones ${ }^{\{12\}}$. Dans les communautés modérément francophones, cette 
proportion diminue à 3.4 médecins francophones par 1000 personnes francophones, tandis que dans les communautés fortement francophones, cette proportion devient 1.3 médecins francophones par 1000 personnes francophones \{12\}. Donc, les moindres taux de médecins francophones furent observés dans les communautés les plus francophones en milieux ruraux de la province, celles qui en nécessitent le plus. Il n'est donc pas surprenant que la santé des francophones soit moins bonne, plus particulièrement en milieu rural, les gens souffrant surtout sans accès à un médecin pouvant communiquer dans leur langue maternelle. Ce n'est ni plus, ni moins, la réalité crue vécue par cette communauté : les ressources pour les Franco-Ontariens en milieu minoritaire sont des plus amoindries.

\section{Propositions palpables d'ajustement}

Évidemment, l'atteinte à l'équité linguistique dans les services de soins de santé au Canada, surtout pour les communautés nordiques de l'Ontario, est un dilemme qu'il faut redresser dans les plus brefs délais ${ }^{\{1\}}$. Afin de rehausser la vitalité des communautés francophones et d'authentifier la garantie bilingue auprès de la population canadienne, plusieurs actions sont nécessaires au niveau fédéral, législatif et communautaire. On y retrouve: 1) l'augmentation de la présence francophone au cœur de groupes décisionnels concernant les politiques francophones, 2) le soutien financier de la part du gouvernement canadien et des ministères de la santé envers des organismes communautaires francophones et 3) le relèvement de la recherche sur la santé des francophones en situation minoritaire.

1) Augmenter la présence francophone dans les groupes décisionnels

En premier lieu, il est important de corriger le manque de présence francophone au sein de groupes décisionnels concernant l'offre de services aux francophones. En effet, les groupes décisionnels, surtout ceux en charge des services francophones, sont majoritairement composés $\mathrm{d}^{\prime}$ anglophones ${ }^{\{13\}}$. Ceci signifie que la présence minoritaire des francophones devient facilement ignorée, et que très peu de progrès ne sont accomplis dans le but d'atteindre un statut de dualité linguistique au Canada ${ }^{113\}}$. Lors d'une entrevue personnelle avec France Gélinas, députée provinciale du Nickel Belt et membre du parti Néo-démocrate, Mme Gélinas reconnaît que les réseaux de santé en français ont éprouvé beaucoup de difficultéspour améliorer les services francophones à cause de leur manque de pouvoir décisionnel ${ }^{[14\}}$. Les propositions de nouveaux programmes et services innovateurs axés sur les besoins des francophones demeuraient ignorés puisque $90 \%$ des dirigeants étaient anglophones et n'avaient pas été sensibilisés aux besoins des francophones ${ }^{[14\}}$. Ici, une voix francophone parmi dix n'est simplement pas suffisante, et le temps est venu pour mieux représenter la communauté francoontarienne ${ }^{\{14\}}$.

Afin d'améliorer l'offre de services francophones en milieu où ils sont minoritaires, les membres des conseils et de l'administration des services de santé en français devrait provenir de la communauté francophone elle-même. Idéalement, les groupes décisionnels de services de langue française devraient être constitués entièrement de franco-ontariens. Tel qu'observé lors du changement de gouvernance des conseils scolaires en Ontario durant l'an 1997, le gouvernement remet l'entière gestion des services éducationnels pour les Franco-Ontariens aux Franco-Ontariens ${ }^{\{15\}}$. En confiant la gouvernance des services de santé francophones aux francophones, il serait finalement possible de répondre congrûment à leurs besoins uniques et de leur ainsi assurer un meilleur état de santé.

2) Engagement financier fédéral envers des organismes communautaires francophones

Afin de combler la disparité entre l'état de santé des francophones et celui des anglophones, l'engagement financier fédéral est d'une importance capitale envers de nombreuses initiatives communautaires francophones qui 
encouragent la disponibilité de services en français ${ }^{22}$. Ces initiatives sont essentielles puisqu'elles visent à améliorer la qualité des services de santé en français, un effort rassurant pour de nombreux Franco-Ontariens malheureusement accoutumés à la présente pénurie de services en français. Dans le plan d'action du Bureau des langues officielles, le gouvernement déclare reconnaître que le manque d'investissements envers les organismes communautaires de langue française hors Québec a créé plusieurs enjeux majeurs. Malgré ce fait, le taux de financement de telles initiatives n'a pas augmenté depuis 2003, rendant difficile une réponse adéquate aux besoins des communautés francophones $\mathrm{du}$ nord de l'Ontario ${ }^{\{16\}}$.

En guise d'exemple, la Société Santé en français (SSF), entité nationale qui vise à améliorer la santé des communautés francophones et acadiennes minoritaires au Canada, opère à travers ses réseaux provinciaux \{17\}. Le mandat du Réseau du mieux-être francophone du Nord de l'Ontario, l'un des 16 réseaux de la Société Santé en français, répond aux besoins de la communauté francophone dans le nord de la province. En plus de la planification de services de santé en français, le réseau est aussi chargé de soumettre des propositions aux autorités gouvernementales au nom des francophones qui vivent en situation minoritaire

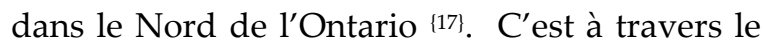
soutien de ces réseaux qu'il est possible d'avoir accès à des centres de santé communautaires francophones, tels que les centres de santé en Ontario. Ces centres fournissent une gamme de services et programmes de promotion de la santé dans 56 communautés en Ontario. De plus, ils sont gérés par des pourvoyeurs de santé francophones qui prennent en considération la culture, la langue, l'éducation, les facteurs économiques, le style de vie, et l'environnement lorsqu'ils fournissent des soins en français ${ }^{118\}}$. Cette initiative conduit vers une améliorationde la qualité des soins, d'autant plus les FrancoOntariens ont aussi tendance à faire plus confiance en leurs pourvoyeurs de services de santé $\{2\}$.

Toutefois, en raison de la perte récente du commissaire aux services en français, les réseaux sont dorénavant les porte-paroles pour la communauté francophone en Ontario. Sans soutien financier gouvernemental adéquat, il leur est impossible d'offrir les services nécessaires afin d'adresser et de répondre aux besoins pressants de la population francophone en situation minoritaire ${ }^{\{2\}}$.

En termes réels, l'engagement financier envers la santé des francophones établi par le gouvernement en 2003 a vu son pouvoir d'achat se dévaloriser au cours des années. Effectivement, la somme totale des budgets est demeurée constante, sans tenir compte de l'inflation, ni des besoins croissants de la population. Il devient alors impossible de réussir des projets d'envergure comme à l'année 2003, partiellement en raison des effets de l'inflation. Récemment, le gouvernement fédéral a établi un plan stratégique, promettant un montant ambitieux de 2,2 milliards de dollars étendus, somme allouée sur une période de cinq ans à l'éducation et aux services gouvernementaux en français ${ }^{\{16\}}$. Néanmoins, ces plans demeurent encore parmi ces nombreuses bonnes initiatives qui ne se sont pas concrétisées en actions à ce jour. Ainsi, l'implication financière gouvernementale est impérative afin de soutenir les organismes francophones, car sans cet appui, la santé des francophones risque d'en souffrir en continuité.

\section{3) Engagement financier fédéral envers de futures recherches}

Il est aussi important que le gouvernement finance la recherche quant à la santé des francophones, indispensable au rehaussement de la qualité et l'accès des services de soins de santé pour les Franco-Ontariens, et ainsi pour conscientiser la population générale au sujet de ce problème accablant ${ }^{22}$. En premier lieu, il faut convaincre le gouvernement que le facteur linguistique des services de santé est primordial, ce qui pourrait encourager l'avancement de plusieurs études de haut calibre au sujet de la 
santé des francophones au Canada, menant ainsi à une meilleure planification de services de santé adaptés à la population francophone. À cet effet, Mme Gélinas déclare, "Ça ne prend pas de temps de se rendre compte que faire des études sur la santé des francophones est extrêmement difficile parce que la plupart du temps, on ne tient pas compte de la langue dans le système de santé. Donc, c'est très difficile de voir, par exemple, si les francophones ont plus de problèmes de santé mentale que les anglophones. En ne tenant pas compte de ces données, on ne peut même pas qualifier la grandeur du problème. On s'en doute, on a des études de moindre envergure qui nous pointent dans la bonne direction, mais le temps avance. Ce n'est pas difficile d'ajouter le facteur langue à notre carte santé de ceux qui veulent s'identifier. Il y a de plus en plus de francophones qui seraient prêts à s'identifier si on leur explique pourquoi" ${ }^{\prime 14\}}$.

D'ailleurs, le gouvernement et les gestionnaires du système de santé doivent également allouer plus de financement aux chercheurs qui s'intéressent à la santé précaire des francophones, déjà limités par la carence de données concernant la santé des FrancoOntariens. Des lors, les chercheurs font face à un sérieux manque de soutien financier par rapport à leurs efforts de recherche. Pourtant, les données probantes pouvant découler de leurs recherches serviront de base solide pour assurer une mise en œuvre efficace de plans concrets. Par exemple, une étude menée en 1989 sur les besoins de la population francophone a accéléré l'ouverture du Centre de santé communautaire du Grand Sudbury ${ }^{\{18\}}$. Mais les obstacles mentionnés ci-dessus peuvent sérieusement entraver tout effort de recherche sur la santé des francophones. Ainsi, l'encouragement et le soutien gouvernemental envers de futures recherches demeure salutaire afin de s'assurer de la visibilité des besoins des populations minoritaires, pour qu'ils cessent d'être ignorés.

Un exemple illustrant la piètre situation de la recherche sur les francophones en situation minoritaire demeure le fait que seulement une des 38 demandes de recherche sur la santé en français au Canada fut financée en $2018{ }^{\text {[19\}. Les }}$ recherches sur la santé en français hors Québec ne reçoivent qu'environ $100000 \$$ de financement, comparativement à la moyenne des subventions envers de projets de recherche généraux, qui eux reçoivent $740000 \${ }^{19}$. Le financement infime accordé aux études menées sur la santé des communautés francophones hors Québec est franchement inadéquat. Il devient presque impossible d'arriver à des solutions lorsque la subvention n'est simplement pas présente pour permettre l'émergence et l'avancement d'études pouvant mener à des pistes concrètes pour résoudre le problème qui perdure. D'après Éric Chevaucherie, gestionnaire de recherche au sein de l'Association des collèges et universités de la francophonie canadienne (ACUFC):

«Ça risque d'entraîner une perte de connaissances sur la santé des communautés francophones en contexte minoritaire alors qu'on sait que la langue est un facteur important dans la santé, notamment pour la santé des aînés ou en santé mentale. Ça menace aussi la formation des professionnels de la santé en français qui n'auront plus de recherches sur lesquelles s'appuyer $»\{19\}$.

La langue, une partie de la culture, est un déterminant important de la santé des francophones qu'il faut considérer et non sousestimer. Les francophones ont des besoins qui diffèrent de la population majoritaire, des besoins uniques et distincts qui méritent d'être redressés lorsqu'ils reçoivent des services de santé ${ }^{\{18\}}$. L'inégalité linguistique qui existe présentement dans les services de soins de santé pose un risque majeur pour les francophones, et pourrait mener à des effets encore plus désastreux si elle demeure à l'écart'11. C'est un problème auquel il faut remédier de façon urgente afin de contrer les répercussions néfastes infligées aux communautés francophones en Ontario.

\section{Impact des propositions sur la pratique et la recherche}


Il existe plusieurs limites qui risquent de ralentir l'exécution des propositions précédentes. Assurément, l'amélioration de l'état de santé des francophones au nord de l'Ontario dépend d'une meilleure collaboration fédérale, provinciale et communautaire ${ }^{[2\}}$. Mais ceci n'est pas la réalité que nous témoignons aujourd'hui à cause de la présence anglophone prédominante parmi les groupes décisionnels influents décisionnels. De ce fait, tenter de convaincre les anglophones de l'importance des services de santé en français n'est pas une tâche simple. Ceux ne sont pas familiers et ne perçoivent pas l'angoisse souvent vécue par les francophones, qui doivent non seulement affronter une situation de crise de santé mais doivent aussi faire face à une barrière linguistique qui devient rapidement insurmontable ${ }^{\{20\}}$. C'est un problème qui s'amplifie lors d'événements catastrophiques, à l'instar de la pandémie du COVID-19 que nous sommes en train de braver présentement.

Ce n'est pas un secret que toute crise accentue et détériore la réalité des communautés minoritaires, tout comme celle des FrancoOntariens, surtout au niveau de la santé mentale. L'accès aux services de santé mentale est pitoyable, et les services de santé mentale ne deviennent que doublement déplorables pour les francophones ${ }^{\{14\}}$. Il s'ajoute le confinement, les pertes d'emplois et le manque de ressources francophones dans les établissements en santé publique, pour s'aligner vers le désastre ${ }^{\{14\}}$. Tout ceci souligne l'importance d'assurer une composition francophone majoritaire ou complète des groupes décisionnels concernant les services de santé en français en Ontario. D'après Mme Gélinas: "par et pour les francophones, c'est la façon d'avancer" ${ }^{\{14\}}$.

Il est inévitable que les changements proposés ci-dessus soient rattachés à des coûts financiers significatifs. En somme, il est contestable que le soutien financier fédéral envers des organismes francophones et de futures recherches sur la santé des francophones soit perçu comme étant dispendieux. Quoiqu'il en soit, c'est une obligation qui, si toujours ignorée, pourrait créer des séquelles encore plus néfastes au système entier. Donc, sans le soutien financier immédiat de la part du gouvernement, la santé des communautés francophones risque d'en souffrir d'autant plus dans les années à venir.

Conséquences possibles reliées à l'inaction face à la problématique

Sans se faire emporter par les quelques obstacles discutés ci-dessus, il faut être conscient que la simple ignorance du problème ne va que mener à des conséquences encore plus sévères. L'une des répercussions les plus importantes est reliée à l'impact direct sur la santé des francophones. Les barrières linguistiques affectent grandement la qualité des services de santé. Elles augmentent les temps de consultation, le nombre de tests diagnostiques requis, et la probabilité d'erreurs diagnostiques [2]. Bien plus, le simple fait que les francophones continuent à éviter de se procurer des services de santé lorsque nécessaire mettra cette population en danger d'aggraver davantage leur état de santé. En effet, comme conséquence dépendre davantage du système de santé, les francophones continueront de se sentir dépourvus, isolés, et incompris ${ }^{221\}}$. Une étude menée auprès de plusieurs communautés nordiques de l'Ontario révèle que la discordance linguistique mène effectivement à de nombreuses conséquences sur la santé des francophones ${ }^{222\}}$ (p.12-33). Les patients francophones sans accès à un pourvoyeur de soins francophone rapportent qu'ils se sentent vulnérables, stressés, et qu'ils ne se sentent pas bienvenus lors des visites chez leur médecin ${ }^{222\}}$ (p.12-33). S'ils ne comprennent pas l'information qui leur est présentée, ils s'abstiennent de poser des questions par crainte de se faire percevoir moins intelligents $\{22\}$ (p.12-33). De surcroît, ceci augmente le niveau de stress supplémentaire pour les francophones et risque $d$ 'induire un sentiment $\mathrm{d}^{\prime}$ insatisfaction et de désarroi ${ }^{\{21\}}$.

Rappelons que la communauté francophone risque de subir des effets cumulatifs d'assimilation au restant de la population [2]. Le concept de complétude institutionnelle, qui 
représente le fait que de détenir une institution contribue directement à l'épanouissement d'une minorité, renforce cette déclaration \{23\} (p.3-14). L'inaction de la part du gouvernement et des autorités du système de santé envers la contrariété des barrières linguistiques auprès des francophones continue à miner la confiance des Franco-Ontariens envers ceux-ci ${ }^{22\}}$. Dans ce cas, il ne s'agit pas du simple droit à des services bureaucratiques en français, mais du bien-être physique et mental de la communauté francophone.

Impact de l'inaction sur la pratique professionnelle en soins de santé

Finalement, la pratique professionnelle risque elle aussi de ressentir les effets du manque de ressources linguistiques pour les communautés francophones au nord de l'Ontario. L'ignorance au sujet des barrières linguistiques dans les services de santé peut nuire non seulement aux francophones ou encore faire souffrir le système de santé, mais également préjudicier les professionnels en soins de santé. À vrai dire, la présence de barrières linguistiques signifie que les services de santé offerts ne sont

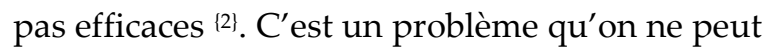
pas se permettre de gérer, vu la réalité courante de ressources restreintes dans le système de santé $\{2\}$.

Les professionnels de la santé sont conscients des effets de barrières linguistiques sur la qualité de leurs services de santé et sur leur engagement professionnel. Sans intervention, les pourvoyeurs de services de santé en français sont à risque de surcharge, ce qui pourrait même causer l'augmentation de l'absentéisme chez eux [2\}. Bref, le fardeau lié aux barrières linguistiques n'affecte pas seulement la population francophone elle-même, mais touche aussi indirectement les professionnels de la santé qui se sentent découragés face au dilemme ${ }^{\{2\}}$.

\section{Ce qui reste à faire pour progresser vers une justice linguistique}

Afin d'atteindre un statut de justice linguistique, il est nécessaire de concrétiser un plan d'action vigoureux avec la collaboration de la communauté francophone. Conscientiser le gouvernement et la population majoritaire au sujet des barrières linguistiques n'est simplement pas suffisant, et il est venu le temps de mettre fin aux plansinopérants en les mettant en action. Les changements proposés dans cet article ne seront possibles que lorsque le reste de la population parviendra à être convaincu de l'importance de ce phénomène.

Tel que démontré lors du mouvement "SOS Montfort", la population franco-ontarienne est apte à créer une résistance politique souveraine lorsque réunie. Comme Mme Gélinas déclare: “C'est à nous, les francophones, de continuer de se faire entendre, de s'organiser et de parler d'une voix de ce que l'on veut vraiment" ${ }^{\text {14\}. }}$ Ainsi, il faut considérer les différentes approches qui pourront mener vers de meilleurs services de santé pour cette communauté.

Comme point de départ, il faut considérer le lobbying auprès de diverses institutions, telles que les réseaux régionaux, les unités sanitaires, ainsi que les hôpitaux à travers du nord de l'Ontario. C'est à l'aide de rassemblements, de conférences de presse et de campagnes publicitaires, qu'on augmentera nos chances d'attirer l'attention des autorités et de pousser le gouvernement de finalement apporter des changements qui vont améliorer la pauvre réalité courante des francophones.

Le lobbying auprès d'institutions éducationnelles est une initiative également importante à considérer. Les services de santé s'étendent à une gamme de domaines et de professions et, il est important que les futurs professionnels de la santé et pourvoyeurs de soins prennent conscience de la situation des francophones en situation minoritaire, euxmêmes étant indirectement affectés par la présence de barrières linguistiques. Il est donc avantageux de les inclure au mouvement avec le but de les sensibiliser à la situation précaire des francophones et aussi dans l'espoir qu'ils soient plus aptes à offrir des services de santé de qualité 
aux Franco-Ontariens, aux bons endroits et selon les besoins.

D'ailleurs, il est nécessaire de pousser les organismes qui s'affichent faussement en tant $\mathrm{qu}^{\prime}$ institutions bilingues de s'approprier correctement leur désignation. La plupart des organismes de services de santé qui s'identifient en tant qu'établissement bilingue n'offrent véritablement pas de services francophones de qualité, et il est temps de les inciter à corriger ce problème. Ici, il serait question de révoquer leur titre d'institution bilingue, ou de les obliger d'adapter leurs services de santé offerts afin de mieux desservir la communauté francophone. D'ailleurs, l'inaccessibilité aux services en français dans les institutions dites "bilingues" est réellement honteuse, et l'ignorance déshonorante du gouvernement ne fait qu'empirer la situation. Afin de pallier aux désavantages mentionnés, une mise à jour de la Loi sur les services en français est absolument nécessaire. Celle-ci énoncera précisément la base des services en français que doivent offrir les diverses institutions à travers la province, et devra aussi dresser les conséquences auxquelles feront face ceux qui continuent de désobéir à la loi. Il n'est pas question d'inventer de nouvelles politiques au sujet de la justice linguistique, mais plutôt d'obliger les politiciens de respecter et de mettre à jour les lois qui existent déjà à ce sujet.

\section{Conclusion}

Les barrières linguistiques continuent d'être un problème irréfutable quant aux soins de santé des francophones en situation minoritaire en Ontario. Ce texte se veut un signal d'alarme, en accentuant fortement la nécessité d'obtenir une collaboration fédérale, provinciale et communautaire, afin de relever l'état de santé précaire des Franco-Ontariens. Il est absolument essentiel de considérer ces facteurs dans la prestation de services et de mieux promouvoir la langue et la culture française dans les milieux où la population est minoritaire. La gouvernance des services par et pour les francophones est l'ultime solution afin d'éviter la dilution des services en français et pour mieux répondre aux besoins pressants de cette communauté unique.

Finalement, le financement fédéral envers des organismes communautaires et des futures recherches sur la santé des Franco-Ontariens est indispensable pour l'avancement des services intolérables présentement offerts à cette population. Sans doute, la lutte des francophones contre l'injustice linguistique, ici au nord de l'Ontario, en est une qui persiste toujours. Pourtant, nous gardons l'espoir qu'un jour, ce peuple pourra finalement atteindre le statut de véritable dualité linguistique, un traitement équitable, tout comme joindre le niveau et l'état de santé caractéristiques aux autres Canadiens.

\section{Remerciements}

Remerciements à Patrick Timony, assistant de recherche au Centre de recherche en santé dans les milieux ruraux et du nord, pour sa contribution et ses suggestions valables lors de la rédaction de cet article. Remerciements abondants à Mme. France Gélinas, députée provinciale de Nickel Belt et membre du parti Néo-démocrate, pour son consentement et sa participation à une entrevue personnelle.

\section{Références}

\{1\}.Forgues, É., Bahi, B., Michaud, J., Deveau, K., Boudreau, J., St-Onge, S. (2011).L'offre de services de santé en français en contexte francophone minoritaire. ICRML. Moncton: NB

\{2\}.Boileau, F. (2009). Special Report on French Language Health Services in Ontario. Office of the French Language Services Commissioner. Toronto: ON

\{3\}.Gouvernement de l'Ontario (2019). Profil de la population francophone de l'Ontario -2016. Repéré de :https://www.ontario.ca/fr/page/profil-de-lapopulation-francophone-de-lontario-2016 
\{4\}.Commissariat aux langues officielles (2018). Pour mieux comprendre vos droits en matière de langues officielles. Toronto: $\mathrm{ON}$

\{5\}.Dupuis, S. (2018). French Language Services Act. The Canadian Encyclopedia.

Repéréde:https://www.thecanadianencyclopedia. ca/en/article/french-language-services-act

\{6\}.Hôpital Montfort (2020). L'histoire de l'Hôpital Montfort.Repéré

de:https://hopitalmontfort.com/fr/lhistoire-delhopital-montfort

\{7\}.Gagnon, M-A. (2017). SOS Montfort. L'encyclopédie canadienne. Toronto: $\mathrm{ON}$

\{8\}.Benoit, M., Bouchard, L., Leis, A. \& Garceau, M.-L. (2012). Les inégalités sociales de santé affectant les communautés francophones en situation minoritaire au Canada. Reflets, 18 (2), 10-18. https://doi.org/10.7202/1013171ar

\{9\}.Branch, S. (2019). L'ultime rapport du commissaire Boileau:Le Droit. Repéré de: https://www.ledroit.com/actualites/francophonie/1 ultime-rapport-du-commissaire-boileau0ef11aeedad257c9701151206f4df126

\{10\}.Gouvernement du Canada (2019). Services gouvernementaux en français. Repéré de: https://www.ontario.ca/fr/page/servicesgouvernementaux-en-francais

\{11\}.Guignard Noël, J., LeBlanc, J., Forgues, É. (2016). Portrait des services bilinguesofferts au sein des hôpitaux canadiens: Rapport. ICRML. Moncton: NB

\{12\}.Timony P, Gauthier A, Hogenbirk JC, Wenghofer E. (2013). Promising quantities, disappointing distribution. Investigating the presence of Frenchspeaking physicians in Ontario's rural Francophone communities. Rural and RemoteHealth2013; 13: 2543.

\{13\}.Radio-Canada (2006). La santé des francophones aux francophones. Repéré de: $\quad$ https://ici.radiocanada.ca/amp/305108/rliss-francophones

\{14\}.Gélinas, F. (2020). Entrevue personnelle.

\{15\}.Sylvestre, P-F. (2007). Douze conseils scolaires de langue française sont créés.L'Express. Repéré de: https://1-express.ca/douze-conseils-scolairesde-langue-francaise-sont-crees/

\{16\}.Gouvernement du Canada (2018). Plan d'action pour les langues officielles -2018-2023 : Investir dans notre avenir. Repéré de: https://www.canada.ca/fr/patrimoinecanadien/services/langues-officiellesbilinguisme/plan-action-langues-officielles/20182023.html

\{17\}.Réseau du mieux-être francophone du Nord de
l'Ontario (2018). Société santé en français. Repéré de: https://www.reseaudumieuxetre.ca/propos/societe -sante-en-francais/

$\{18\}$.Centre de santé communautaire du Grand Sudbury (2018). Historique. Repéré de: https://santesudbury.ca/

$\{19\}$.Vachet, B. (2018). La recherche en santé en français menacée:ONfr+. Repéré de: https://onfr.tfo.org/la-recherche-en-sante-enfrancais-menacee/

$\{20\}$.Bouchard, L., Gaboury, I., Chomienne, M-H., Gilbert, A., Dubois, L. (2009). La santé en situation linguistique minoritaire. Politiques de Santé. NCBI. Repéré de: https://www.ncbi.nlm.nih.gov/pmc/articles/ PMC2700700/

\{21\}.Bowen, S., de Moissac, D. (2018). Impact of language barriers on quality of care and patient safety for official language minority Francophones in Canada. Repéré de: https://journals.sagepub.com/doi/full/10.1177/237 $\underline{4373518769008}$

\{22\}.Jutras, C., Gauthier, A., Timony, P., Kpazaï, G. (2020). Expérience de francophones en Ontario chez leur médecine de famille : concordance et discordance linguistique. Revue de la Diversité de la Recherche en Santé. DRHJ/RDRS 2020, 3, pp.12-33

\{23\}.Cardinal, L., \& Léger, R. (2017). La complétude institutionnelle en perspective.Politiques et Sociétés (Vol. 36, pp. 3-14). Société québécoise de science politique.Montréal:QC.doi: https://doi.org/10.7202/1042233ar 